\title{
Optimal Waveform Design for Dual-functional MIMO Radar-Communication Systems
}

\author{
Longfei Zhou*, Fan Liu ${ }^{\dagger}$, Chang Tian*, Christos Masouros ${ }^{\ddagger}$, Ang Li ${ }^{\ddagger}$, Wei Jiang*, Wu Luo* \\ *State Key Laboratory of Advanced Optical Communication Systems and Networks, Peking University, Beijing, P.R. China, \\ Email:\{zhoulongfei,tianch,jiangwei,luow\}@pku.edu.cn \\ ${ }^{\dagger}$ School of Information and Electronics, Beijing Institute of Technology, Beijing, P.R. China \\ $\ddagger$ Department of Electronic and Electrical Engineering, University College London, London, UK
}

\begin{abstract}
This paper considers the waveform design for dual-functional multi-input-multi-output (MIMO) radarcommunication systems. Two optimization-based novel waveform designs are proposed. The aim of the first waveform design is to minimize the downlink multi-user interference (MUI) energy by exploiting the remaining degrees of freedom (DoFs) while always guaranteeing the radar performance to be optimal. The second waveform design is a trade-off optimization between radar and communication performances by allowing a tolerable mismatch between the designed and the desired radar beampatterns. Albeit non-convexity of both problems, efficient algorithms are devised to obtain globally optimal solutions, which can be used for simultaneous target detection and downlink communications. Numerical results show that the communication performance could be significantly improved by tolerating a slight radar performance loss and therefore a favorable balance between communication and radar performances is achievable.
\end{abstract}

\section{INTRODUCTION}

Frequency spectrum is one of the most valuable resources for wireless communication. Realizing the scarcity of the spectrum, network providers and policy regulators are exploring the feasibility to share with LTE and Wi-Fi systems in the near future the spectrum that is currently occupied by other applications [1]-[4], such as airborne radars and navigation systems close to the $3.4 \mathrm{GHz}$ band [5] and shipborne and Vessel Traffic Service (VTS) radar at 5.6 GHz [6]. As an emerging research topic, the communication-radar spectrum sharing (CRSS) not only presents the advantage for enabling the efficient usage of the spectrum, but also provides a new way for designing novel systems that can benefit from the cooperation of radar and communication.

A naive way to achieve the spectral coexistence between communication and radar systems is opportunistic spectrum sharing [7]. Nevertheless, it does not allow both systems to work simultaneously. In view of this, many works [8][13] considered the null-space projection (NSP) scheme to different spectral coexistence scenarios between radar and communication systems, in which a radar beamformer is designed to project the signals onto the null-space of the interference channel between the radar and base station (BS)/user equipment (UE), such that the interference from the radar to the communication link is zero. However, this leads to performance loss for the radar, since the beamforming is no longer optimal for target detection and estimation. More recent contributions [14]-[18] have exploited optimization techniques to achieve trade-offs between the performances of radar and communication by relaxing the zero-forcing constraint of precoder to impose controllable interference levels on the communication systems [9], offering a more realistic coexistence.

A critical drawback of above coexistence approaches is that radar and communication devices are typically required to exchange side information for achieving a beneficial cooperation, such as channel state information, radar probing waveforms and communication modulation formats. This results in considerable extra complexity to the system, and is therefore difficult to implement in practical scenarios. The novel dualfunctional radar-communication (RadCom) system has been regarded as a favorable CRSS approach to avoid this shortfall. As an enabling solution, dual-functional waveform design can support target detection and information transmission at the same time. Such possibilities have been explored for single-antenna systems [19]-[22], where several integrated waveforms have been proposed to combine the radar and communication signals. Further, [23], [24] considered waveform shuffling across the antennas or Phase Shift Keying (PSK) by different beamformer weighting factors as the communication modulation schemes. A common feature of these methods is that one communication symbol is represented by one or several radar pulses, which leads to a low data rate in the order of the radar pulse repetition frequency (PRF).

The contributions of this work are two optimization-based novel waveform designs for dual-functional MIMO RadCom systems. First, we consider the waveform design by minimizing the downlink MUI under certain radar-specific constraints that guarantee the radar performance to be optimal. Next, we consider the optimization of trade-off between radar and communication performances under a power budget constraint. Although non-convex, both problems are efficiently solved. It is worth highlighting that the proposed methods yield provably globally optimal waveforms, which can be used for simultaneous target detection and downlink communications. Simulation results show that the trade-off optimization can achieve a good balance between communication and radar performances.

Notation: Lowercase and uppercase boldface letters denote column vectors and matrices, respectively. Operators $\operatorname{Tr}(\cdot)$, $(\cdot)^{\mathrm{T}},(\cdot)^{\mathrm{H}}$ and $\|\cdot\|_{\mathrm{F}}$, correspond to the trace, the transpose, 


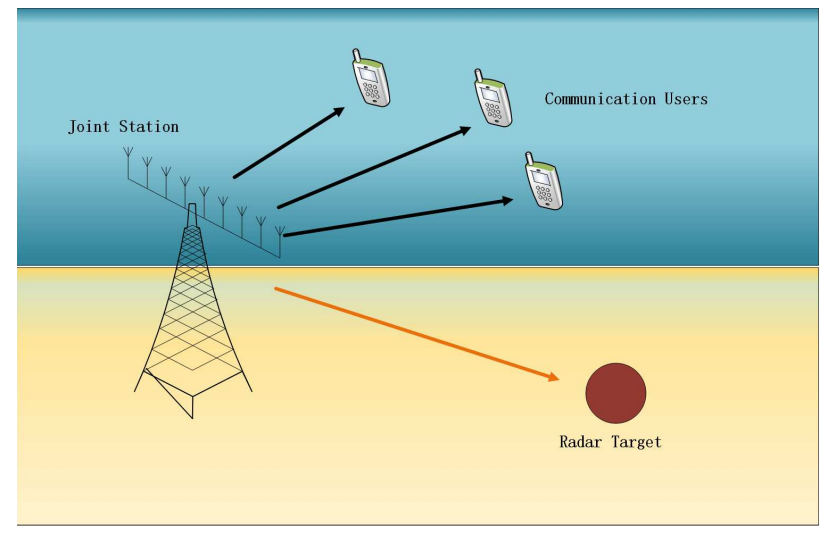

Fig. 1. Illustration of a joint MIMO radar-communication system.

the conjugate transpose, the Frobenius norm operations, respectively. $\operatorname{Re}(\cdot)$ denotes the real part of complex numbers. $\mathbf{x} \sim \mathcal{C N}(\mathbf{m}, \mathbf{C})$ means that random vector $\mathbf{x}$ is circularlysymmetric complex Gaussian with mean $\mathbf{m}$ and covariance C.

\section{SySTEM Model}

We consider a joint MIMO radar-communication system as shown in Fig.1, which simultaneously transmits radar probing waveform to the targets and communication symbols to the downlink users. The joint station is equipped with a uniform linear array (ULA) with $N$ antennas, serving $K$ single-antenna users while detecting radar targets at the same time.

\section{A. MIMO communication model}

The received signals at the downlink users is given by

$$
\mathbf{Y}=\mathbf{H X}+\mathbf{W}
$$

where $\mathbf{H}=\left[\mathbf{h}_{1}, \mathbf{h}_{2}, \ldots \mathbf{h}_{K}\right]^{\mathrm{T}} \in \mathbb{C}^{K \times N}$ is the channel matrix, $\mathbf{X}=\left[\mathbf{x}_{1}, \mathbf{x}_{2}, \ldots, \mathbf{x}_{L}\right] \in \mathbb{C}^{N \times L}$ is the transmitted signal matrix with $L$ being the length of the communication frame, and $\mathbf{W}=\left[\mathbf{w}_{1}, \mathbf{w}_{2}, \ldots, \mathbf{w}_{L}\right] \in \mathbb{C}^{K \times L}$ is the noise matrix with $\mathbf{w}_{j} \sim \mathcal{C N}\left(0, N_{0} \mathbf{I}_{N}\right)$ for all $1 \leq j \leq L$.

We assume [25]: 1) Dual-functional waveform is the transmitted signal matrix $\mathbf{X}$ for both radar and communication operations. In this case, each communication symbol is also a snapshot of a radar subpulse; 2) The channel $\mathbf{H}$ is flat Rayleigh fading, and remains unchanged during one communication frame/radar subpulse; 3 ) The channel $\mathbf{H}$ is perfectly estimated by pilot symbols.

Given the desired symbol matrix $\mathbf{S} \in \mathbb{C}^{K \times L}$ for the downlink users, the received signals can be rewritten as

$$
\mathbf{Y}=\mathbf{S}+\underbrace{(\mathbf{H X}-\mathbf{S})}_{\text {MUI }}+\mathbf{W} \text {. }
$$

For each user, entries of $\mathbf{S}$ are assumed to be drawn from the same constellation. The second term in (2) represents the multiuser interference. The total MUI energy is used as the figure of merit of communication, which is expressed as

$$
P_{\mathrm{MUI}}=\|\mathbf{H X}-\mathbf{S}\|_{\mathrm{F}}^{2} .
$$

The MUI energy is a key performance measure since it is closely related to the achievable sum-rate of the downlink users [26]. Specifically, the achievable sum-rate is

$$
R=\sum_{i=1}^{K} \log _{2}\left(1+\gamma_{i}\right),
$$

where $\gamma_{i}$ is the signal-to-interference-plus-noise ratio (SINR) per frame for the $i$-th user, which is given by

$$
\gamma_{i}=\frac{\mathbb{E}\left(\left|s_{i, j}\right|^{2}\right)}{\mathbb{E}\left(\left|\mathbf{h}_{i}^{\mathrm{T}} \mathbf{x}_{j}-s_{i, j}\right|^{2}\right)+N_{0}} .
$$

Here, $s_{i, j}$ is the $(i, j)$-th entry of $\mathbf{S}$, and $\mathbb{E}(\cdot)$ denotes the ensemble average with respect to the time index. For a given constellation with fixed energy, the power of useful signal $\mathbb{E}\left(\left|s_{i, j}\right|^{2}\right)$ is also fixed. Hence, the sum-rate can be maximized through minimization of the MUI energy.

\section{B. MIMO radar model}

Traditional phased-array radar transmits over each antenna correlated signals, indeed, different phase-shifted versions of a common waveform. In contrast, MIMO radar employs uncorrelated waveforms and results in higher DoFs than the traditional phased-array radar. The existing literature [27], [28] indicate that the design of radar beampattern is equivalent to designing the covariance matrix of the probing signals. Spatial covariance matrix of the dual-functional waveform matrix $\mathbf{X}$ is expressed as

$$
\mathbf{R}_{X}=\frac{1}{L} \mathbf{X X}^{\mathrm{H}}
$$

Without loss of generality, we assume $L \geq N$ to ensure positive definiteness of $\mathbf{R}_{X}$. Further, the transmit beampattern for the RadCom system can be characterized as

$$
P_{d}(\theta)=\mathbf{a}(\theta)^{\mathrm{H}} \mathbf{R}_{X} \mathbf{a}(\theta),
$$

where $\mathbf{a}(\theta)=\left[1, e^{j 2 \pi \Delta \sin (\theta)}, \ldots, e^{j 2 \pi(N-1) \Delta \sin (\theta)}\right]^{\mathrm{T}} \in \mathbb{C}^{N}$ is the steering vector of the ULA with $\theta$ being the detection angle and $\Delta$ being the spacing between adjacent antennas normalized by the wavelength.

\section{Problem Formulation And Optimal Design}

Two optimization problems for dual-functional MIMO RadCom systems in terms of $P_{\mathrm{MUI}}$ and $\mathbf{R}_{X}$, are formulated in the following.

\section{A. Waveform optimization under radar beampattern con- straints}

We first consider the minimization of MUI energy under MIMO radar-specific constraints. Given a covariance matrix $\mathbf{R}_{d}$ that corresponds to a well-designed MIMO radar beampattern, the MUI minimization problem is expressed as

$$
\begin{array}{ll}
\min _{\mathbf{X}} & \|\mathbf{H X}-\mathbf{S}\|_{\mathrm{F}}^{2} \\
\text { s.t. } & \frac{1}{L} \mathbf{X X}^{\mathrm{H}}=\mathbf{R}_{d},
\end{array}
$$


where $\mathbf{R}_{d}$ is a Hermitian positive-definite matrix satisfying $\operatorname{Tr}\left(\mathbf{R}_{d}\right)=P_{T}$, and $P_{T}$ is the total transmit power. The case of $\mathbf{R}_{d}=\frac{P_{T}}{N} \mathbf{I}_{N}$ corresponds to the omni-directional beampattern design, which is usually used in MIMO radar for initial probing. While a general $\mathbf{R}_{d}$ with $\operatorname{Tr}\left(\mathbf{R}_{d}\right)=P_{T}$ corresponds to a specific beampattern design that points to the directions of interest, which is used for tracking after target detection.

Problem (8) is non-convex due to the nonlinear equality constraint $(8 b)$. Fortunately, the problem could be efficiently solved to global optimality by utilizing the special structure.

Proposition 1. Given the Cholesky decomposition of $\mathbf{R}_{d}=$ $\mathbf{F F}^{H}$ and a singular value decomposition (SVD) of $\mathbf{F}^{H} \mathbf{H}^{H} \mathbf{S}=$ $\mathbf{U} \boldsymbol{\Sigma} \mathbf{V}^{H}$, where $\mathbf{F}$ is invertible and $\mathbf{U} \in \mathbb{C}^{N \times N}$ and $\mathbf{V} \in$ $\mathbb{C}^{L \times L}$ are unitary. A globally optimal solution $\mathbf{X}_{*}$ to (8) is

$$
\mathbf{X}_{*}=\sqrt{L} \mathbf{F} \mathbf{U V} \mathbf{V}_{N}^{H},
$$

where $\mathbf{V}_{N}$ is the first $N$ columns of $\mathbf{V}$.

Proof: Let $\mathbf{Z}=(\sqrt{L} \mathbf{F})^{-1} \mathbf{X}$. Substituting it in (8b) gives $\mathbf{Z Z}^{\mathrm{H}}=\mathbf{I}_{N}$. We can rewrite (8a) as

$$
\begin{aligned}
& \|\mathbf{H X}-\mathbf{S}\|_{\mathrm{F}}^{2}=\operatorname{Tr}\left((\sqrt{L} \mathbf{H F Z}-\mathbf{S})(\sqrt{L} \mathbf{H F Z}-\mathbf{S})^{\mathrm{H}}\right) \\
& =\operatorname{Tr}\left(L \mathbf{H F} \mathbf{F}^{\mathrm{H}} \mathbf{H}^{\mathrm{H}}\right)-2 \sqrt{L} \operatorname{Re}\left(\operatorname{Tr}\left(\mathbf{S Z}^{\mathrm{H}} \mathbf{F}^{\mathrm{H}} \mathbf{H}^{\mathrm{H}}\right)\right)+\operatorname{Tr}\left(\mathbf{S S}^{\mathrm{H}}\right) .
\end{aligned}
$$

By the cyclic property of trace operation, solving (8) is equivalent to the following problem

$$
\max _{\mathbf{Z}}\left\{\operatorname{Re}\left(\operatorname{Tr}\left(\mathbf{Z}^{\mathrm{H}} \mathbf{F}^{\mathrm{H}} \mathbf{H}^{\mathrm{H}} \mathbf{S}\right)\right): \mathbf{Z} \mathbf{Z}^{\mathrm{H}}=\mathbf{I}_{N}\right\} .
$$

Let $\mathbf{T}=\mathbf{V}^{\mathrm{H}} \mathbf{Z}^{\mathrm{H}} \mathbf{U}$, it follows that $\mathbf{T}^{\mathrm{H}} \mathbf{T}=\mathbf{I}_{N}$ from the unitarity of $\mathbf{V}$ and $\mathbf{U}$. By the SVD of $\mathbf{F}^{\mathrm{H}} \mathbf{H}^{\mathrm{H}} \mathbf{S}$, we obtain

$$
\begin{aligned}
\operatorname{Re}\left(\operatorname{Tr}\left(\mathbf{Z}^{\mathrm{H}} \mathbf{F}^{\mathrm{H}} \mathbf{H}^{\mathrm{H}} \mathbf{S}\right)\right) & =\operatorname{Re}\left(\operatorname{Tr}\left(\mathbf{Z}^{\mathrm{H}} \mathbf{U} \boldsymbol{\Sigma} \mathbf{V}^{\mathrm{H}}\right)\right) \\
& =\operatorname{Re}\left(\operatorname{Tr}\left(\mathbf{V}^{\mathrm{H}} \mathbf{Z}^{\mathrm{H}} \mathbf{U} \boldsymbol{\Sigma}\right)\right) \\
& =\operatorname{Re}(\operatorname{Tr}(\mathbf{T} \boldsymbol{\Sigma})) \\
& =\operatorname{Re}\left(\sum_{i=1}^{N} T_{i, i} \Sigma_{i, i}\right) .
\end{aligned}
$$

By $\mathbf{T}^{\mathrm{H}} \mathbf{T}=\mathbf{I}_{N}$, we have $\left|T_{i, j}\right| \leq 1 \forall i, j$. Hence, a maximizer to the above sum is $\mathbf{T}_{*}=\mathbf{I}_{L \times N}$, which is an $L \times N$ matrix composed by an $N \times N$ identity matrix and an $(L-N) \times N$ zero matrix. Therefore, by definition of $\mathbf{T}$ and $\mathbf{Z}$, a globally optimal solution to (8) is $\mathbf{X}_{*}=\sqrt{L} \mathbf{F} \mathbf{U} \mathbf{I}_{N \times L} \mathbf{V}^{\mathrm{H}}=$ $\sqrt{L} \mathbf{F} \mathbf{U V}_{N}^{\mathrm{H}}$.

\section{B. Trade-off between radar and communication performances}

It should be highlighted that in problem (8) the radar performance is guaranteed to be optimal under the covariance constraint (8b) and the MUI energy is minimized by exploiting the remaining DoFs. When the remaining DoFs is low, the communication counterpart may suffer from serious performance loss. We therefore consider a trade-off design by allowing a tolerable mismatch between the designed and the desired radar beampatterns. Denoting an obtained optimal solution to (8) by $\mathbf{X}_{0}$, the trade-off problem can be set up in the following form

$$
\begin{array}{ll}
\min _{\mathbf{X}} & \rho\|\mathbf{H X}-\mathbf{S}\|_{\mathrm{F}}^{2}+(1-\rho)\left\|\mathbf{X}-\mathbf{X}_{0}\right\|_{\mathrm{F}}^{2} \\
\text { s.t. } & \frac{1}{L}\|\mathbf{X}\|_{\mathrm{F}}^{2}=P_{T} .
\end{array}
$$

where $0 \leq \rho \leq 1$ is a weighting factor that makes compromises between radar and communication performances.

Two additional remarks for above formulation are given as follows. First, for ensuring the coherence between (8) and (12), we enforce an equality constraint for the power budget, as the radar station is often required to transmit at its maximum available power in practice. Second, it seems at the first sight that a more natural formulation is to use $\left\|\mathbf{X X}^{\mathrm{H}}-L \mathbf{R}_{d}\right\|_{\mathrm{F}}^{2}$ rather than $\left\|\mathbf{X}-\mathbf{X}_{0}\right\|_{\mathrm{F}}^{2}$ in (12a). The price is the intractability of the resulting quartic polynomial optimization problem.

Problem (12) is a non-convex quadratic program with only one quadratic constraint, and indeed matrix version of the trust-region subproblem (TRS) [29]. Hence, the strong duality holds [30], i.e., the duality gap is zero. Furthermore, according to [30], the semidefinite programming relaxation (SDR) for (12) is tight, i.e., the SDR admits a rank-one solution, which yields a globally optimal solution to (12). Nevertheless, due to the large number of variables in the problem, SDR is not computationally efficient for (12). Hence, we propose a lowcomplexity algorithm that achieves the global optimum in the following.

Let $\mathbf{X}_{*}$ and $\lambda_{*}$ be a pair of optimal primal and dual solutions. Sufficient and necessary global optimality conditions for (12) are given as [30]

$$
\begin{aligned}
\left(\mathbf{Q}+\lambda_{*} \mathbf{I}_{N}\right) \mathbf{X}_{*} & =\mathbf{G}, \\
\left\|\mathbf{X}_{*}\right\|_{\mathrm{F}}^{2} & =L P_{T}, \\
\mathbf{Q}+\lambda_{*} \mathbf{I}_{N} & \succeq 0,
\end{aligned}
$$

where $\mathbf{Q}=\rho \mathbf{H}^{\mathrm{H}} \mathbf{H}+(1-\rho) \mathbf{I}_{N}$ and $\mathbf{G}=\rho \mathbf{H}^{\mathrm{H}} \mathbf{S}+(1-\rho) \mathbf{X}_{0}$. Conditions (13a), (13b) and (13c) guarantee stationarity, primal and dual feasibility, respectively. Conditions (13a) and (13b) are the well-known Karush-Kuhn-Thcker (KKT) conditions for local optimality, while the condition (13c) excludes the non-global optimal stationary solutions.

From (13c), we have $\lambda_{*} \geq-\lambda_{N}$, where $\lambda_{N}$ is the minimum eigenvalue of $\mathbf{Q}$. We will deal with two cases separately: (a) $\lambda_{*}=-\lambda_{N}$ or (b) $\lambda_{*}>-\lambda_{N}$. The case of $\lambda_{*}=-\lambda_{N}$ corresponds to the 'hard case' in trust region literature, which is unstable and rarely occurs in practice. It is easy to check by matrix decomposition whether equation (13a) for $\lambda_{*}=-\lambda_{N}$ is consistent and admits a solution $\mathbf{X}_{*}$ satisfying $\left\|\mathbf{X}_{*}\right\|_{\mathrm{F}}^{2}=$ $L P_{T}$ if consistent.

We next focus on the case of $\lambda_{*}>-\lambda_{N}$. It follows from (13a) that

$$
\begin{aligned}
\mathbf{X}_{*}\left(\lambda_{*}\right) & =\left(\mathbf{Q}+\lambda_{*} \mathbf{I}_{N}\right)^{-1} \mathbf{G} \\
& =\mathbf{V}\left(\Lambda+\lambda_{*} \mathbf{I}_{N}\right)^{-1} \mathbf{V}^{\mathrm{H}} \mathbf{G},
\end{aligned}
$$


Algorithm 1 Low-complexity Algorithm for Solving (12)

Input: $\mathbf{H}, \mathbf{S}, \mathbf{X}_{0}, P_{T}$, and weighting factor $\rho \in(0,1)$.

Output: $\epsilon$-optimal solution $\mathbf{X}_{*}$.

1. Compute $\mathbf{Q}, \mathbf{G}$ and the eigenvalue decomposition of $\mathbf{Q}$.

2. Check whether (13a) for $\lambda_{*}=-\lambda_{N}$ admits a solution

$\mathbf{X}_{*}$ satisfying (13b). If failed, go to Step 3.

3. Find an $\epsilon$-optimal solution $\lambda_{*}$ to (13b) over $\left(-\lambda_{N}, \lambda_{u b}\right)$ by bisection search and compute $\mathbf{X}_{*}=\left(\mathbf{Q}+\lambda_{*} \mathbf{I}_{N}\right)^{-1} \mathbf{G}$.

where $\mathbf{Q}=\mathbf{V} \Lambda \mathbf{V}^{\mathrm{H}}$ is the eigenvalue decomposition of $\mathbf{Q}$ and $\Lambda=\operatorname{diag}\left(\lambda_{1}, \lambda_{2}, \ldots, \lambda_{N}\right)$. We obtain

$$
\begin{aligned}
\left\|\mathbf{X}_{*}\left(\lambda_{*}\right)\right\|_{\mathrm{F}}^{2} & =\sum_{i=1}^{N} \sum_{j=1}^{L} \frac{\left|\left[\mathbf{V}^{\mathrm{H}} \mathbf{G}\right]_{i, j}\right|^{2}}{\left(\lambda_{*}+\lambda_{i}\right)^{2}} \\
& \leq \frac{N L}{\left(\lambda_{*}+\lambda_{N}\right)^{2}} \max _{i, j}\left|\left[\mathbf{V}^{\mathrm{H}} \mathbf{G}\right]_{i, j}\right|^{2} .
\end{aligned}
$$

By (15a), function $\left\|\mathbf{X}_{*}\left(\lambda_{*}\right)\right\|_{\mathrm{F}}^{2}$ is strictly decreasing and convex on the interval $\lambda_{*}>-\lambda_{N}$. Thus, equation $\left\|\mathbf{X}_{*}\left(\lambda_{*}\right)\right\|_{\mathrm{F}}^{2}=$ $L P_{T}$ has a unique root $\lambda_{*}$, i.e., the optimal dual solution. By (13b) and (15b), an upper bound of $\lambda_{*}$ is given by

$$
\lambda_{*} \leq-\lambda_{N}+\sqrt{\frac{N}{P_{T}}} \max _{i, j}\left|\left[\mathbf{V}^{\mathrm{H}} \mathbf{G}\right]_{i, j}\right| \triangleq \lambda_{u b} .
$$

Therefore, the unique optimal dual solution $\lambda_{*}$ can be solved by root-finding methods for $\left\|\mathbf{X}_{*}\left(\lambda_{*}\right)\right\|_{\mathrm{F}}^{2}=L P_{T}$ on the interval $\left(-\lambda_{N}, \lambda_{u b}\right), e . g$., bisection search or Ridders' method [31]. The rate of convergence of bisection search or Ridders' method is at least linear, which means that the algorithms reach an $\epsilon$-optimal solution within $\mathcal{O}(\log (1 / \epsilon))$ iterations. The computation burden is dominated by the one-pass eigenvalue decomposition of $\mathbf{Q}$ and matrix multiplication of $\mathbf{V}^{\mathrm{H}} \mathbf{G}$, which cost $\mathcal{O}\left(N^{3}\right)$ and $\mathcal{O}\left(N^{2} L\right)$ arithmetic operations, respectively. For clarity, overall solution procedure for (12) is summarized in Algorithm 1.

\section{Simulation Results}

In this section, we present numerical results to assess the proposed waveform design approaches. The simulation settings are as follows. Assume that the station is equipped with a ULA with half-wavelength spacing between the adjacent antennas and each entry of the channel matrix $\mathbf{H}$ is standard Complex Gaussian, i.e., $h_{i, j} \sim \mathcal{C N}(0,1)$. Unit-power QPSK alphabet is chosen as the constellation for communication, i.e., the power of each entry in the symbol matrix $\mathbf{S}$ is one. For simplicity, we set $P_{T}=1, N=64$ and $L=70$, and define SNR $=P_{T} / N_{0}$. Symbols 'Omni' and 'Directional' are used to represent the omni-directional beampattern design and the directional beampattern design, respectively. Further, the waveform designs based on (8) and on (12) are denoted by 'Strict' and 'Tradeoff', respectively.

Fig. 2 and Fig. 3 show the average achievable rate and the associated radar beampatterns obtained by different approaches for $K=30$, respectively. User's average achievable rates are

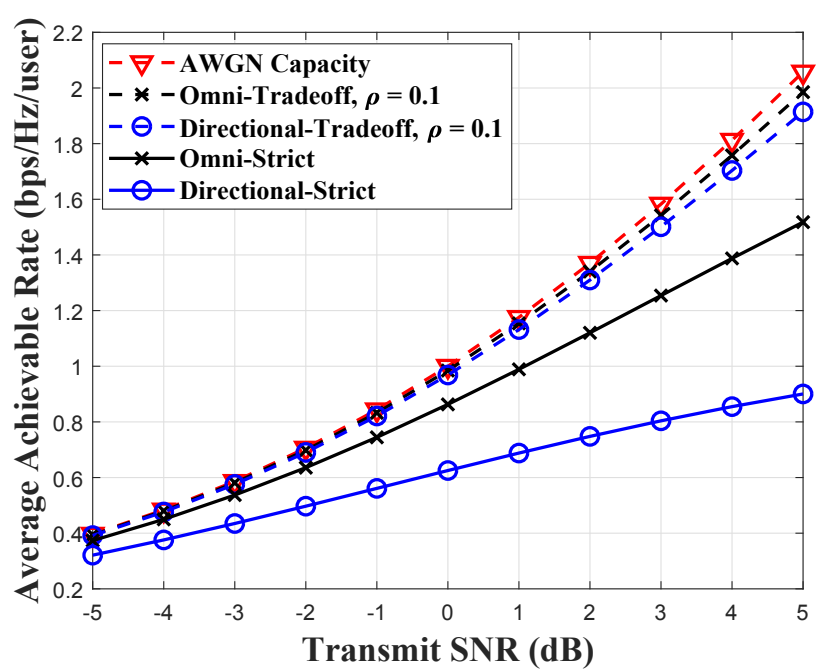

Fig. 2. Average achievable rate versus transmit SNR for $K=30$.

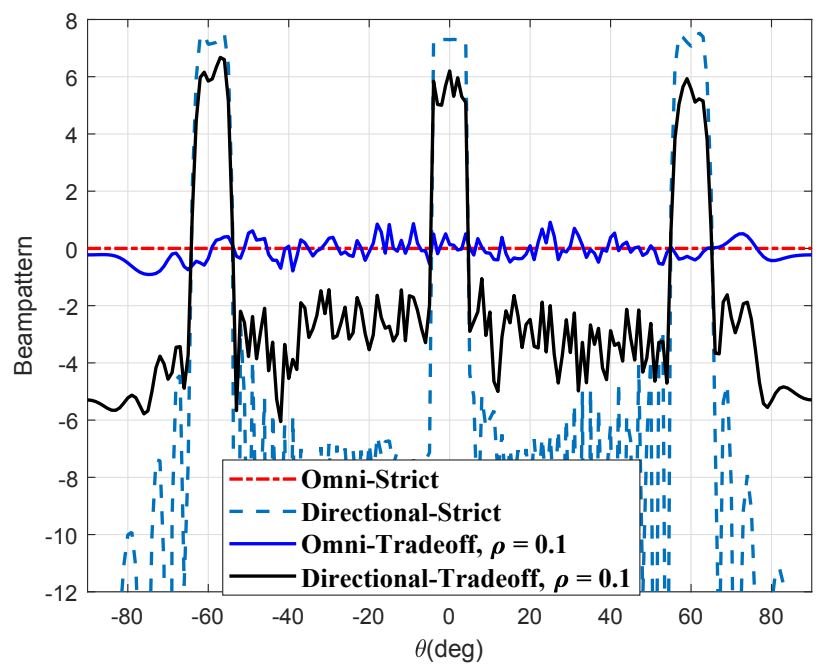

Fig. 3. Radar beampatterns obtained by different approaches $(\mathrm{K}=30)$.

computed by (4) and (5) and the AWGN channel capacity is calculated as $\log _{2}(1+\mathrm{SNR})$. We consider three targets of interest with angles of $-60^{\circ}, 0^{\circ}, 60^{\circ}$ for directional beampattern design, and utilize the classic least-squares techniques [32] to obtain the desired covariance matrix $\mathbf{R}_{d}$ as defined in (8). It can be seen from Fig.2 that the 'Strict' designs result in a relatively serious performance loss of communication and average achievable rates can be increased significantly by the 'Trade-off' designs, which are close to the AWGN channel capacity without MUI. Meanwhile, we observe from Fig.3 that the radar beampatterns of the 'Trade-off' designs with weighting factor $\rho=0.1$, only experience a slight performance loss of radar, compared with the 'Strict', i.e., the desired optimal beampatterns. Specifically, the performance loss is less than $3 \mathrm{~dB}$ around angles of the targets of interest.

Fig. 4 and Fig.5 show the trade-off curves between the 


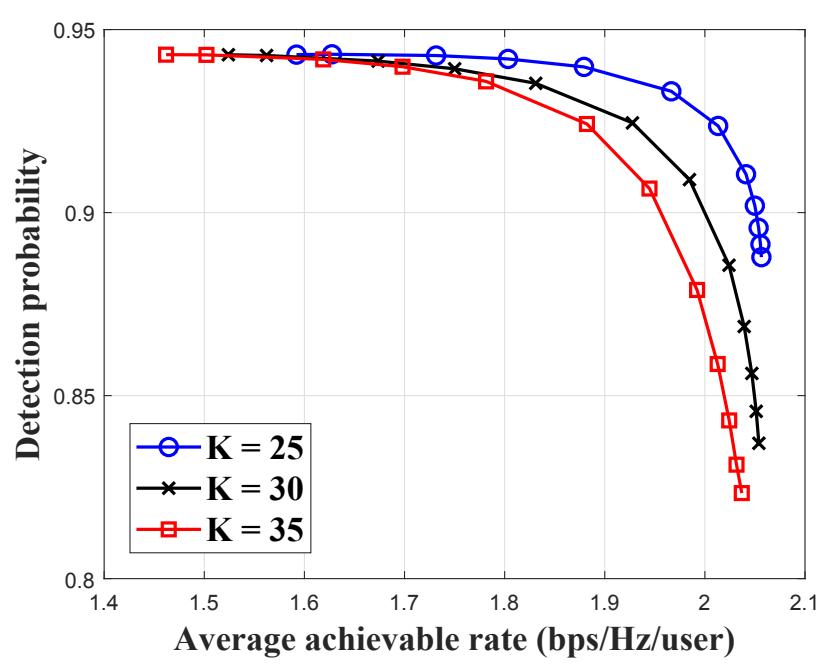

Fig. 4. Trade-off of omni-directional beampattern design between the user's average achievable rate and the radar detection probability.

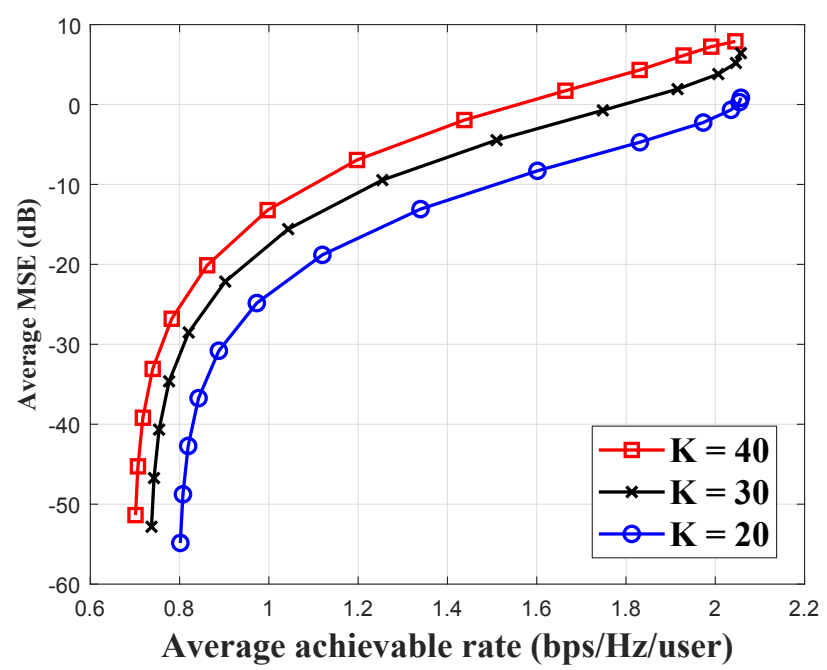

Fig. 5. Trade-off of directional beampattern design between the user's average achievable rate and the radar average MSE.

communication and radar performances by sweeping the weighting factor $\rho$ over $(0,1)$. Detection probability, which is calculated based on [11, eq. (69)], is used as the metric for omni-directional beampattern design, where we consider the constant false-alarm rate (CFAR) detection for a point-like target in the far field, located at the angle of $36^{\circ}$. The received SNR is fixed at $-19 \mathrm{~dB}$ and the false-alarm probability for radar is $10^{-7}$. Mean squared error (MSE) between the desired and obtained beampatterns is used as the radar metric for directional beampattern design. It can be seen from Fig.4 and Fig. 5 that there exists a trade-off between communication rate and radar detection/estimation performance. The average achievable rate increases for the fixed detection probability or MSE, as the number of users decreases, which suggests that the MUI energy can be further minimized by increasing the DoFs. Both figures indicate that the trade-off waveform design can achieve a favorable balance between the communication and radar performances.

\section{CONCLUSION}

In this paper, we discuss the waveform design for dualfunctional MIMO radar-communication system, which can be used for both target detection and downlink communications. Two optimization-based novel waveform designs are proposed. Efficient algorithms are devised to obtain provable globally optimal solutions. Numerical results show the achievability of a good balance between communication and radar performances.

\section{REFERENCES}

[1] FCC. (2010) Connecting America: The national broadband plan. [Online]. Available: https://www.fcc.gov/general/national-broadband-plan

[2] DARPA. (2016) Shared spectrum access for radar and communications (SSPARC). [Online]. Available: http://www.darpa. $\mathrm{mil} /$ program/shared-spectrum-access-for-radar-and-communications

[3] NSF. (2013) Enhancing access to the radio spectrum (EARS). [Online]. Available: https://www.nsf.gov/pubs/2013/nsf13539/nsf13539.htm

[4] - (2016) Spectrum efficiency, energy efficiency, and security (SpecEES): Enabling spectrum for all. [Online]. Available: https: //www.nsf.gov/pubs/2016/nsf16616/nsf16616.htm

[5] Ofcom. (2015) Public sector spectrum release: Award of the 2.3 and $3.4 \mathrm{GHz}$ spectrum bands. [Online]. Available: https://www.ofcom.org. uk/consultations-and-statements/category-1/2.3-3.4-ghz-auction-design

[6] ECC. (2016) The european table of frequency allocations and applocations in the frequency range $8.3 \mathrm{kHz}$ to $3000 \mathrm{GHz}$ (ECA table). [Online]. Available: http://www.erodocdb.dk/docs/doc98//official/ pdf/ERCREP025.PDF

[7] R. Saruthirathanaworakun, J. M. Peha, and L. M. Correia, "Opportunistic sharing between rotating radar and cellular," IEEE J. Sel. Areas Commun., vol. 30, no. 10, pp. 1900-1910, 2012.

[8] S. Sodagari, A. Khawar, T. C. Clancy, and R. McGwier, "A projection based approach for radar and telecommunication systems coexistence," in 2012 IEEE Global Commun. Conf. (GLOBECOM), Dec 2012, pp. 5010-5014.

[9] A. Babaei, W. H. Tranter, and T. Bose, "A nullspace-based precoder with subspace expansion for radar/communications coexistence," in 2013 IEEE Global Commun. Conf. (GLOBECOM), Dec 2013, pp. 3487-3492.

[10] A. Khawar, A. Abdelhadi, and T. C. Clancy, "On the impact of timevarying interference-channel on the spatial approach of spectrum sharing between S-band radar and communication system," in 2014 IEEE Military Commun. Conf., Oct 2014, pp. 807-812.

[11] A. Khawar, A. Abdelhadi, and C. Clancy, "Target detection performance of spectrum sharing MIMO radars," IEEE Sensors Journal, vol. 15, no. 9, pp. 4928-4940, Sept 2015.

[12] A. Khawar, A. Abdelhadi, and T. C. Clancy, "Coexistence analysis between radar and cellular system in LoS channel," IEEE Antennas Wireless Propag. Lett., vol. 15, pp. 972-975, 2016.

[13] J. A. Mahal, A. Khawar, A. Abdelhadi, and T. C. Clancy, "Spectral coexistence of MIMO radar and MIMO cellular system," IEEE Trans. Aerosp. Electron. Syst., vol. 53, no. 2, pp. 655-668, April 2017.

[14] B. Li and A. Petropulu, "Radar precoding for spectrum sharing between matrix completion based MIMO radars and a MIMO communication system," in 2015 IEEE Global Conf. on Signal and Inform. Process. (GlobalSIP), Dec 2015, pp. 737-741.

[15] B. Li, A. P. Petropulu, and W. Trappe, "Optimum co-design for spectrum sharing between matrix completion based MIMO radars and a MIMO communication system," IEEE Trans. Signal Process., vol. 64, no. 17, pp. 4562-4575, Sept 2016.

[16] B. Li and A. Petropulu, "Joint transmit designs for co-existence of MIMO wireless communications and sparse sensing radars in clutter," IEEE Trans. Aerosp. Electron. Syst., vol. PP, no. 99, pp. 1-1, 2017.

[17] L. Zheng, M. Lops, X. Wang, and E. Grossi, "Joint design of overlaid communication systems and pulsed radars," IEEE Transactions on Signal Processing, vol. 66, no. 1, pp. 139-154, Jan 2018.

[18] L. Zheng, M. Lops, and X. Wang, "Adaptive interference removal for uncoordinated radar/communication coexistence," IEEE Journal of Selected Topics in Signal Processing, vol. 12, no. 1, pp. 45-60, Feb 2018. 
[19] A. R. Chiriyath, B. Paul, G. M. Jacyna, and D. W. Bliss, "Inner bounds on performance of radar and communications co-existence," IEEE Trans. Signal Process., vol. 64, no. 2, pp. 464-474, Jan 2016.

[20] A. R. Chiriyath, B. Paul, and D. W. Bliss, "Radar-communications convergence: Coexistence, cooperation, and co-design," IEEE Trans. on Cognitive Commun. and Networking, vol. 3, no. 1, pp. 1-12, March 2017.

[21] G. N. Saddik, R. S. Singh, and E. R. Brown, "Ultra-wideband multifunctional communications/radar system," IEEE Trans. Microw. Theory Tech., vol. 55, no. 7, pp. 1431-1437, July 2007.

[22] C. Sturm and W. Wiesbeck, "Waveform design and signal processing aspects for fusion of wireless communications and radar sensing," Proc. IEEE, vol. 99, no. 7, pp. 1236-1259, July 2011.

[23] E. BouDaher, A. Hassanien, E. Aboutanios, and M. G. Amin, "Towards a dual-function MIMO radar-communication system," in 2016 IEEE Radar Conference (RadarConf), May 2016, pp. 1-6.

[24] A. Hassanien, M. G. Amin, Y. D. Zhang, and F. Ahmad, "Phasemodulation based dual-function radar-communications," IET Radar, Sonar Navigation, vol. 10, no. 8, pp. 1411-1421, 2016.

[25] F. Liu, C. Masouros, A. Li, H. Sun, and L. Hanzo, "Mu-mimo communications with mimo radar: From co-existence to joint transmission," IEEE Transactions on Wireless Communications, vol. 17, no. 4, pp. 2755-2770, April 2018.

[26] S. K. Mohammed and E. G. Larsson, "Per-antenna constant envelope precoding for large multi-user MIMO systems," IEEE Trans. Commun., vol. 61, no. 3, pp. 1059-1071, March 2013.

[27] J. Li and P. Stoica, "MIMO radar with colocated antennas," IEEE Signal Process. Mag., vol. 24, no. 5, pp. 106-114, 2007.

[28] — MIMO Radar Signal Processing. Wiley-IEEE Press, 2008.

[29] R. J. Stern and H. Wolkowicz, "Indefinite trust region subproblems and nonsymmetric eigenvalue perturbations," SIAM Journal on Optimization, vol. 5, no. 2, pp. 286-313, 1995.

[30] C. Fortin and H. Wolkowicz, "The trust region subproblem and semidefinite programming," Optimization methods and software, vol. 19, no. 1, pp. 41-67, 2004.

[31] C. Ridders, "A new algorithm for computing a single root of a real continuous function," IEEE Trans. Circuits Syst., vol. 26, no. 11, pp. 979-980, 1979.

[32] D. R. Fuhrmann and G. S. Antonio, "Transmit beamforming for MIMO radar systems using signal cross-correlation," IEEE Transactions on Aerospace and Electronic Systems, vol. 44, no. 1, pp. 171-186, January 2008. 